Searching for plasmas with anomalous dispersion in the soft $\mathrm{X}$-ray regime

Joseph Nilsen, Walter R. Johnson, K. T. Cheng

August 27, 2007

SPIE Optics and Photonics 2007

San Diego, CA, United States

August 26, 2007 through August 30, 2007 
This document was prepared as an account of work sponsored by an agency of the United States Government. Neither the United States Government nor the University of California nor any of their employees, makes any warranty, express or implied, or assumes any legal liability or responsibility for the accuracy, completeness, or usefulness of any information, apparatus, product, or process disclosed, or represents that its use would not infringe privately owned rights. Reference herein to any specific commercial product, process, or service by trade name, trademark, manufacturer, or otherwise, does not necessarily constitute or imply its endorsement, recommendation, or favoring by the United States Government or the University of California. The views and opinions of authors expressed herein do not necessarily state or reflect those of the United States Government or the University of California, and shall not be used for advertising or product endorsement purposes. 


\title{
Searching for plasmas with anomalous dispersion in the soft X-ray regime
}

\author{
Joseph Nilsen ${ }^{1}$, Walter R. Johnson ${ }^{2}$, and K. T. Cheng ${ }^{1}$ \\ ${ }^{1}$ Lawrence Livermore National Laboratory, Livermore, CA 94551 \\ ${ }^{2}$ University of Notre Dame, Notre Dame, IN 46556
}

\begin{abstract}
Over the last decade the electron density of plasmas has been measured using X-ray laser interferometers in the 14 to 47 $\mathrm{nm}$ wavelength regime. With the same formula used in decades of experiments with optical interferometers, the data analysis assumes the index of refraction is due only to the free electrons, which makes the index less than one. Over the last several years, interferometer experiments in $\mathrm{C}, \mathrm{Al}, \mathrm{Ag}$, and $\mathrm{Sn}$ plasmas have observed plasmas with index of refraction greater than one at 14 or $47 \mathrm{~nm}$ and demonstrated unequivocally that the usual formula for calculating the index of refraction is not always valid as the contribution from bound electrons can dominate the free electrons in certain cases. In this paper we search for other materials with strong anomalous dispersion that could be used in X-ray laser interferometer experiments to help understand this phenomena. An average atom code is used to calculate the plasma properties. This paper discusses the calculations of anomalous dispersion in $\mathrm{Ne}$ and $\mathrm{Na}$ plasmas near $47 \mathrm{~nm}$ and $\mathrm{Xe}$ plasmas near $14 \mathrm{~nm}$. With the advent of the FLASH X-ray free electron laser in Germany and the LCLS X-FEL coming online at Stanford in 2 years the average atom code will be an invaluable tool to explore plasmas at higher X-ray energy to identify potential experiments for the future. During the next decade X-ray free electron lasers and other X-ray sources will be used to probe a wider variety of plasmas at higher densities and shorter wavelengths so understanding the index of refraction in plasmas will be even more essential.
\end{abstract}

Keywords: X-ray laser, Interferometers; Index of refraction; Plasmas; Anomalous dispersion

\section{INTRODUCTION}

From the earliest days of lasers, optical interferometers have been used to measure the electron density of plasmas [1] assuming that the index of refraction of the plasma is due only to the free electrons [1-2]. This assumption makes the electron density of the plasma directly proportional to the number of fringe shifts in the interferometer and further assumes that that the index of refraction in the plasma is always less than one. A decade ago the first X-ray laser interferometer [3] operating at a wavelength of $15.5 \mathrm{~nm}$ was demonstrated at the Nova facility at Lawrence Livermore National Laboratory (LLNL). Over the past decade many X-ray laser interferometers [4-6], as well as a high order harmonic interferometer [7], have been built in the wavelength range of 14 to $72 \mathrm{~nm}$. All the data analysis done with these sources assume only the free electrons contribute to the index of refraction. In the future, interferometers will be built using the VUV and X-ray free electron lasers, which will extend lasers to even shorter wavelengths [8]. 
In the last several years interferometer experiments of $\mathrm{Al}$ plasmas [4,5,9], Sn and $\mathrm{Ag}$ plasmas [10], and $\mathrm{C}$ plasmas [11] have observed fringe lines bend in the opposite direction than was expected, indicating that the index of refraction was greater than one. The traditional analysis of the experiments would indicate that the electron density was less than zero. The original experiments in the Al plasmas were done at the Advanced Photon Research Center at JAERI using the $13.9 \mathrm{~nm}$ Ni-like Ag laser [4] and at the COMET laser facility at LLNL using the $14.7 \mathrm{~nm}$ Ni-like Pd laser [5]. The analysis of all these experiments show that the anomalous dispersion from the resonance lines and absorption edges of the bound electrons have a large contribution to the index of refraction with the opposite sign as the free electrons and this explains how the index of refraction is greater than one in these plasmas [10-14]. Initially, a surprising result of the calculations is that the influence of the bound electrons on the index of refraction extends far from the absorption edges and resonance lines [12]. Resonance lines affect the index of refraction at photon energies located orders of magnitude further from the line centers than the corresponding line widths owing to the fact that they contribute through a dispersion integral.

The original analysis [12] of the index of refraction for Al plasmas was done at a single wavelength, $14.7 \mathrm{~nm}$, by combining individual calculations done for each iso-electronic sequence of Al. That analysis pointed out the need for a new tool that would let us calculate the index of refraction for any plasma at any wavelength. The INFERNO average atom code has been used for many years to calculate the distribution of levels and the absorption coefficient for plasma at a given temperature and density [15]. By modifying this code [16], we are now able to calculate the index of refraction for a wide range of plasma conditions. To validate the average atom code [13] we compared with calculations done with the more detailed OPAL code [17-19].

In this work we search for other materials that can be used to create plasmas with index of refraction greater than one at X-ray laser energies. We discuss how neutral $\mathrm{Na}$ vapor and singly-ionized Ne plasma look to be promising candidates to use in interferometer experiments based on the Ne-like Ar X-ray laser at $26.44 \mathrm{eV}$. For triply-ionized Xe, we present calculations that show that this plasma will have an index of refraction greater than one at the energy of the Pd X-ray laser at $84.46 \mathrm{eV}$.

\section{ANALYSIS OF INTERFEROMETER EXPERIMENTS}

Assuming only free electrons contribute to the index of refraction, the formula for the index of refraction of a plasma is $n=\left(1-\mathrm{N}_{\text {elec }} / \mathrm{N}_{\text {crit }}\right)^{1 / 2}$ where $\mathrm{N}_{\text {elec }}$ is the electron density of the plasma and $\mathrm{N}_{\text {crit }}$ is the plasma critical density. At wavelength $\lambda, \mathrm{N}_{\text {crit }}=\pi /\left(\mathrm{r}_{0} \lambda^{2}\right)$ where $\mathrm{r}_{0}$ is the classical electron radius, $2.818 \times 10^{-13} \mathrm{~cm}$ [2]. In typical experiments the electron density is much less than the critical density so the formula above can be approximated by $n=1-\left(\mathrm{N}_{\text {elec }} / 2 \mathrm{~N}_{\text {crit }}\right)$. For a plasma that is uniform over length $L$ the number of fringe shifts observed in an interferometer equals $(1-n) L / \lambda$. For a non-uniform plasma one does a path length integral. The fringe shifts are referenced against a set of reference fringes in the absence of any plasma and the formula assumes that the interferometer is in a vacuum. Using the approximations described above, the number of fringe shifts equals $\left(\mathrm{N}_{\text {elec }} \quad L\right) /\left(\begin{array}{llll}2 & \lambda & \mathrm{N}_{\text {crit }}\end{array}\right)$. When analyzing an experiment one counts how far the fringes have shifted compared with the reference fringes and converts this into electron density. For the $46.9 \mathrm{~nm}$ Ar X-ray laser the number of fringe shifts in the interferometer is $\left(\mathrm{N}_{\text {elec }} \mathrm{L}\right) /(4.75 \mathrm{x}$ $10^{18} \mathrm{~cm}^{-2}$ ) and the critical density is $5.07 \times 10^{23} \mathrm{~cm}^{-3}$.

For a plasma we can define a complex index of refraction $n^{*}=1-\delta-i \beta$. The real part of the index of refraction $n$ $=1-\delta$. The imaginary part of the index of refraction is due to absorption. The total absorption coefficient $\alpha=N_{\text {ion }} \sigma=$ $(4 \pi \beta) / \lambda$ where $\mathrm{N}_{\text {ion }}$ is the ion density of the plasma and $\beta$ is the imaginary part of the complex index of refraction $n^{*}$. The Henke tables tabulate the dimensional-less optical constants $f_{2}$ and $f_{1}$ for neutral materials. These are related to $\delta$ and $\beta$ by $\delta=f_{1} N_{\text {ion }} /\left(2 N_{\text {crit }}\right)$ and $\beta=f_{2} N_{\text {ion }} /\left(2 N_{\text {crit }}\right)$ [20]. From the total absorption cross-section $\sigma$ we determine the 
optical constant $f_{2}=\sigma /\left(2 \lambda r_{0}\right)$. We then derive the optical constant $f_{1}$ as a function of photon energy $E$ using the Kramers-Kronig dispersion relation [21]. This involves taking the principal value of the integral

$$
f_{1}(E)=Z_{n u c}+\frac{2}{\pi} P . V \cdot \int_{0}^{\infty} \frac{f_{2}(\varepsilon) \varepsilon d \varepsilon}{E^{2}-\varepsilon^{2}}
$$

where $Z_{\text {nuc }}$ is the atomic number of the element. This means we include the total number of bound and free electrons when calculating the dispersion relation. For example, $Z_{\text {nuc }}=11$ for a Na plasma. For neutral materials the oscillator sum rules insure that $f_{1}$ goes to zero at zero energy and $Z_{\text {nuc }}$ at infinite energy. For an ionized plasma with average ionization $\mathrm{Z}^{*}$ then $\mathrm{f}_{1}=\mathrm{Z}^{*}$ at $\mathrm{E}=0$.

In the absence of any bound electrons $f_{1}$ is equivalent to the number of free electrons per ion. This means that we can replace $\mathrm{N}_{\text {elec }}$ with $\mathrm{f}_{1} \mathrm{~N}_{\text {ion }}$ in the formula for the number of fringe shifts. The free electron approximation for the index of refraction is true when $f_{1}$ is equal to $Z^{*}$. The number of fringe shifts observed in the experiment for a real plasma is now equals $\left(f_{1} N_{\text {ion }} L\right) /\left(\begin{array}{lll}2 & \lambda & N_{\text {crit }}\end{array}\right)$. Taking the ratio of $f_{1}$ to $Z^{*}$ gives the ratio of the measured electron density to the actual electron density. When the ratio is negative, the index of refraction is greater than one and the fringes bend the opposite direction than expected in the interferometer.

\section{AVERAGE ATOM CODE}

The INFERNO code [15] has been used for many years to calculate the ionization conditions and absorption spectrum of plasmas under a wide variety of conditions. This code uses the average-atom technique. For finite temperatures and densities, the INFERNO code calculates a statistical population for occupation of one-electron Dirac orbitals in the plasma. We use a non-relativistic version of INFERNO in this work to calculate bound and continuum orbitals and the corresponding self-consistent potential. By applying linear response theory we obtain an average-atom version of the Kubo-Greenwood equation [22,23] for the frequency-dependent conductivity of the plasma. The imaginary part of the complex dielectric function is proportional to the conductivity. The real part of the dielectric function can be found from its imaginary part using a Kramers-Kronig [21] dispersion relation. The details of the KuboGreenwood formula applied to the average-atom model are described in Ref. 16.

The average-atom results [13] have been validated against calculations done with the OPAL code [17-19]. The OPAL code was developed at the LLNL to compute opacities of low- to mid-Z elements. In the OPAL code the plasma is treated as a mixture of atoms in discrete ionization states, thereby giving a more realistic description of individual lines and absorption edges. However the OPAL calculations can only be done for a limited range of ions.

To do the calculation of the optical properties of various plasmas, we assume the plasmas have a constant ion density of $10^{20} \mathrm{~cm}^{-3}$ and we vary the temperature to change the ionization state of the plasma. Since the average atom code was developed to calculate higher density materials near solid density it is presently difficult to calculate at lower densities than $10^{20} \mathrm{~cm}^{-3}$. In most laser plasmas we are typically dealing with plasmas at this density or one to two orders of magnitude lower density. Even though the calculations are done for a single ion density the optical constants $f_{1}$ and $f_{2}$ that we calculate normalize away the actual density so we expect the analysis to be valid over a wide range of densities. The code assume the electron, ion, and radiation temperatures are equal and the plasma is in local thermodynamic equilibrium (LTE). For a dynamic plasma that is not in equilibrium, the ionization condition $\left(Z^{*}\right)$ is a good way to compare plasma rather than the actual temperature of the plasma. $Z^{*}=1$ means the average ion is singly ionized, 2 means double ionized, etc. 


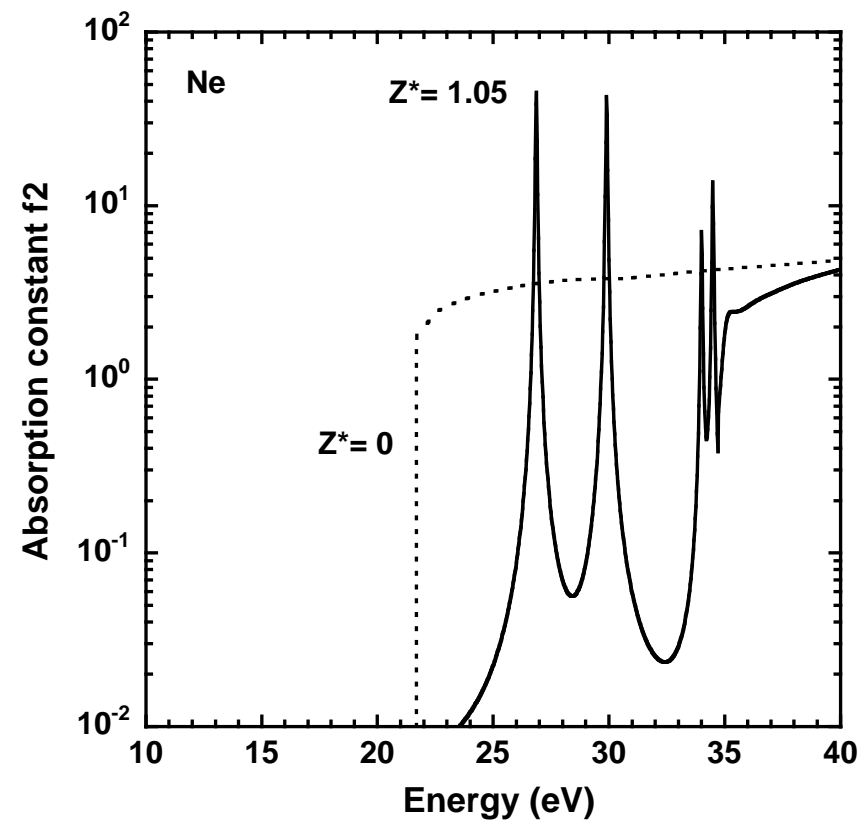

Fig. 1. Absorption constant $f_{2}$ versus photon energy for Ne plasmas. The dotted line is from the Henke data. The solid curve is from the average atom code for $Z^{*}=1.05$. The average ionization state is given by $Z^{*}$. The ion density is $10^{20} \mathrm{~cm}^{-3}$.

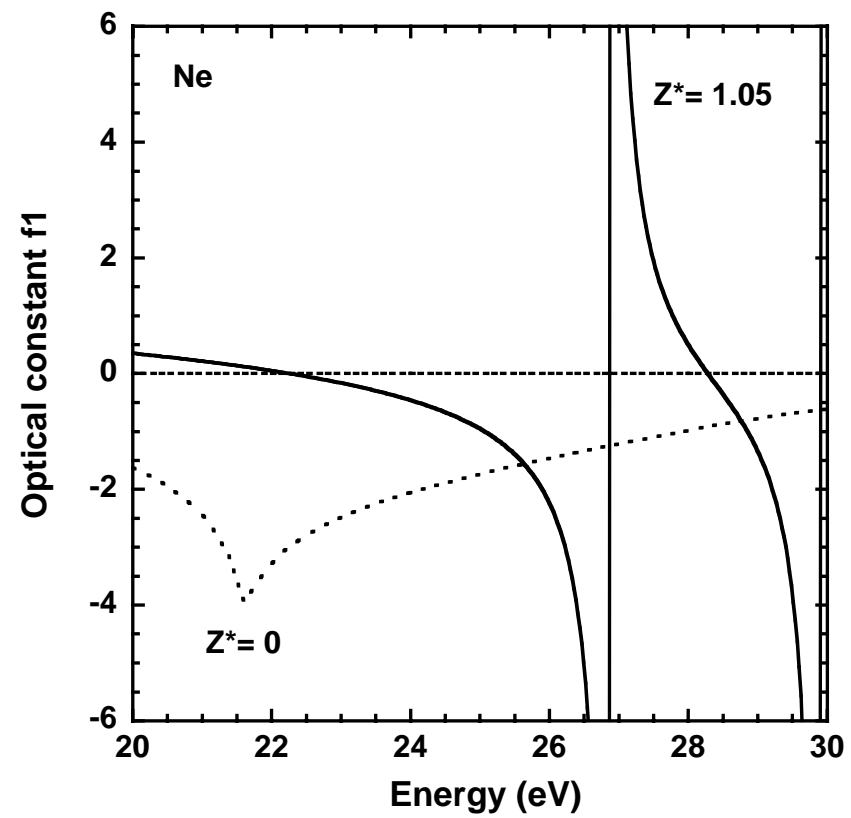

Fig. 2. Optical constant $f_{1}$ versus photon energy for Ne plasmas. The dotted line is from the Henke data. The solid curve is from the average atom code for $Z^{*}=1.05$. The average ionization state is given by $Z^{*}$. The ion density is $10^{20} \mathrm{~cm}^{-3}$. The other dotted line at a value of 0 is a visual aid. 


\section{MODELING OF NEON AND SODIUM PLASMAS AT 46.9 NM}

We have been looking for other plasmas in which we could observe a strong contribution from bound electrons that would make the index of refraction larger than one. For the interferometer that uses the Ne-like Ar X-ray laser at 46.9 $\mathrm{nm}(26.44 \mathrm{eV})$ we first looked at the Henke data to find materials with absorption edges in the $20-30 \mathrm{eV}$ range. Since $\mathrm{Ne}$ and $\mathrm{Na}$ both looked like interesting materials with $\mathrm{L}$ shell edges at 21.6 and $30.6 \mathrm{eV}$, respectively, we extrapolated the Henke data to estimate the $f_{1}$ versus photon energy for neutral $\mathrm{Ne}$ and $\mathrm{Na}$. Finding negative $f_{1}$ values for neutral materials is usually a good clue to find negative $\mathrm{f}_{1}$ in plasma that are only a few times ionized.

For neutral Ne the plasma would be very opaque to the $26.44 \mathrm{eV}$ X-rays since they are above the L-shell absorption edge at $21.6 \mathrm{eV}$. However for single ionized neon the L-edge moves to $41 \mathrm{eV}$ and the plasma becomes much more transparent as shown in Fig. 1 which plots the absorption constant $\mathrm{f}_{2}$ versus photon energy for Ne plasmas. The case $\mathrm{Z}^{*}$ $=0$, shown by the dotted line, is the Henke data. Using an ion density of $10^{20} \mathrm{~cm}^{-3}$ at a temperature of $4 \mathrm{eV}$ the average atom code calculates a $Z^{*}=1.05$, which is close to singly-ionized. This calculated spectrum, shown by the solid line, is shifted by $4.18 \mathrm{eV}$ to higher energy so that the $2 \mathrm{~s}-2 \mathrm{p}$ line agrees with the experimentally measured value of $26.86 \mathrm{eV}$ [24]. Figure 2 shows the optical constant $f_{1}$ versus photon energy for the Ne plasmas. For the case $Z^{*}=0$, shown by the dotted line, the Henke data has been extrapolated to lower energy. At an energy of $26.44 \mathrm{eV} \mathrm{f}_{1}=-1.4$ for the neutral $\mathrm{Ne}$ but this case would be highly absorbing. For the singly ionized case with $Z^{*}=1.05$, shown by the solid line, $\mathrm{f}_{1}$ is now -4.4 at the photon energy of $26.44 \mathrm{eV}$. This is due to being on the low energy side of the strong $2 \mathrm{~s}-2 \mathrm{p}$ absorption line. While not shown in the figure, as one continues to ionize Ne to doubly-ionized the strong $2 s-2 p$ line moves to lower energy at $25.33 \mathrm{eV}$ and the net result is $\mathrm{f}_{1}$ reaches a positive value of 3.9 at the $26.44 \mathrm{eV}$ photon energy. In a real experiment using the Ar X-ray laser one could observe the neutral Ne gas go from opaque to transparent as the gas is ionized and furthermore watch the fringe shifts go from negative to positive as $\mathrm{Ne}$ is ionized from singly to doubly ionized. At some point there would be no fringe shift when there was the right mixture of singly and doubly ionized Ne. One could even envision using the ionization state of $\mathrm{Ne}$ as a switch that could move the Ar X-ray laser from one direction to another.

For $\mathrm{Na}$ we have a slightly simpler situation. Neutral $\mathrm{Na}$ has its L-edge at $30.6 \mathrm{eV}$ and this moves to higher energy as $\mathrm{Na}$ is ionized so $\mathrm{Na}$ starts out quite transparent to the Ar X-ray laser photons at $26.44 \mathrm{eV}$. Fig. 3 shows the absorption constant $f_{2}$ versus photon energy for the two cases with $Z^{*}=0$ (Henke data) and $Z^{*}=2.0$ (average atom code with temperature of $7.3 \mathrm{eV}$ ). The doubly ionized case has the spectrum shifted by $5.5 \mathrm{eV}$ so that the $2 \mathrm{~s}-2 \mathrm{p}$ line agrees with the experimentally measured value of $32.7 \mathrm{eV}$ [25]. If we now plot the optical constant $\mathrm{f}_{1}$ versus photon energy for the two cases above, as show in Fig. 4, $\mathrm{f}_{1}=-0.87$ for neutral $\mathrm{Na}$ and goes to 1.44 for doubly-ionized Na. For singly ionized case the average atom code estimates a value of -0.15 for $\mathrm{f}_{1}$. This suggests that an experiment with neutral Na gas would

observe the anomalous dispersion and the fringe shifts would go through a zero point near singly ionized $\mathrm{Na}$ and eventually look quite normal as one approached doubly-ionized Na. Keep in mind that $f_{1}$ is still not equal to $Z^{*}$ for doubly-ionized $\mathrm{Na}$ so the measured electron density in an actual experiment would still be low by the ratio of $\mathrm{f}_{1}$ to $\mathrm{Z}^{*}$. 


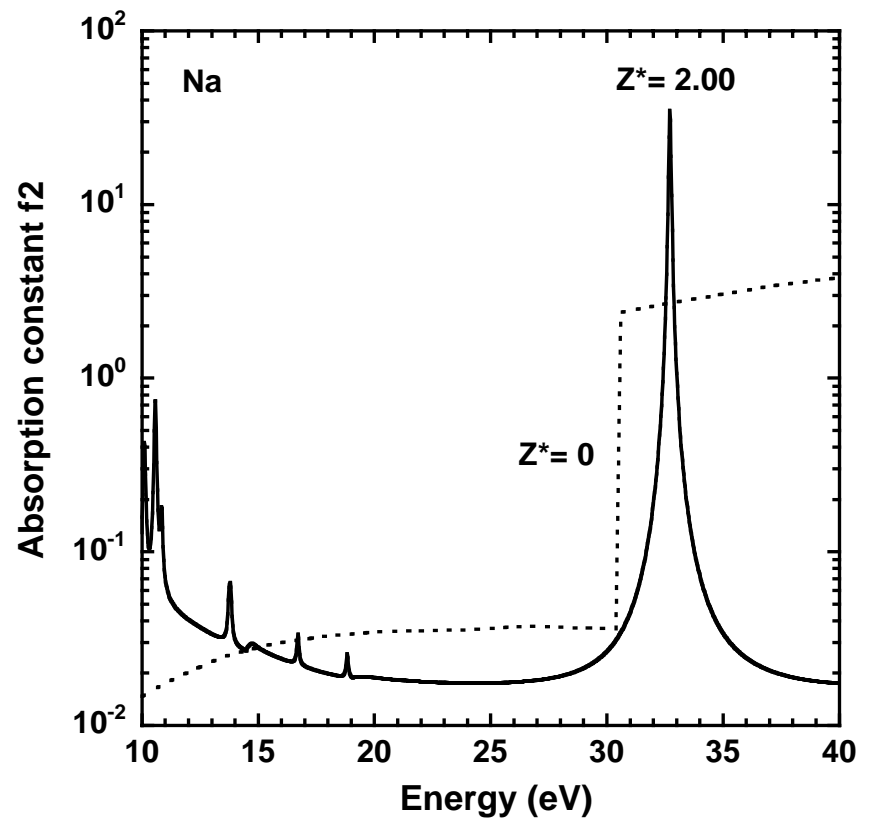

Fig. 3. Absorption constant $f_{2}$ versus photon energy for Na plasmas. The dotted line is from the Henke data. The solid curve is from the average atom code for $Z^{*}=2.0$. The average ionization state is given by $Z^{*}$. The ion density is $10^{20} \mathrm{~cm}^{-3}$.

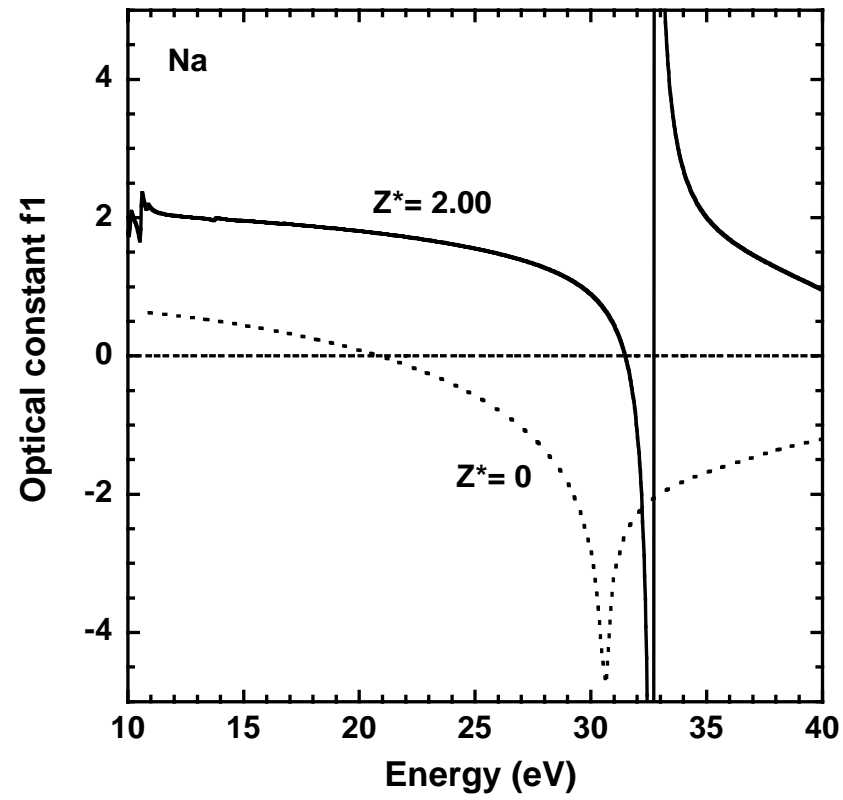

Fig. 4. Optical constant $f_{1}$ versus photon energy for Na plasmas. The dotted line is from the Henke data. The solid curve is from the average atom code for $Z^{*}=2.0$. The average ionization state is given by $Z^{*}$. The ion density is $10^{20} \mathrm{~cm}^{-3}$. The other dotted line at a value of 0 is a visual aid. 


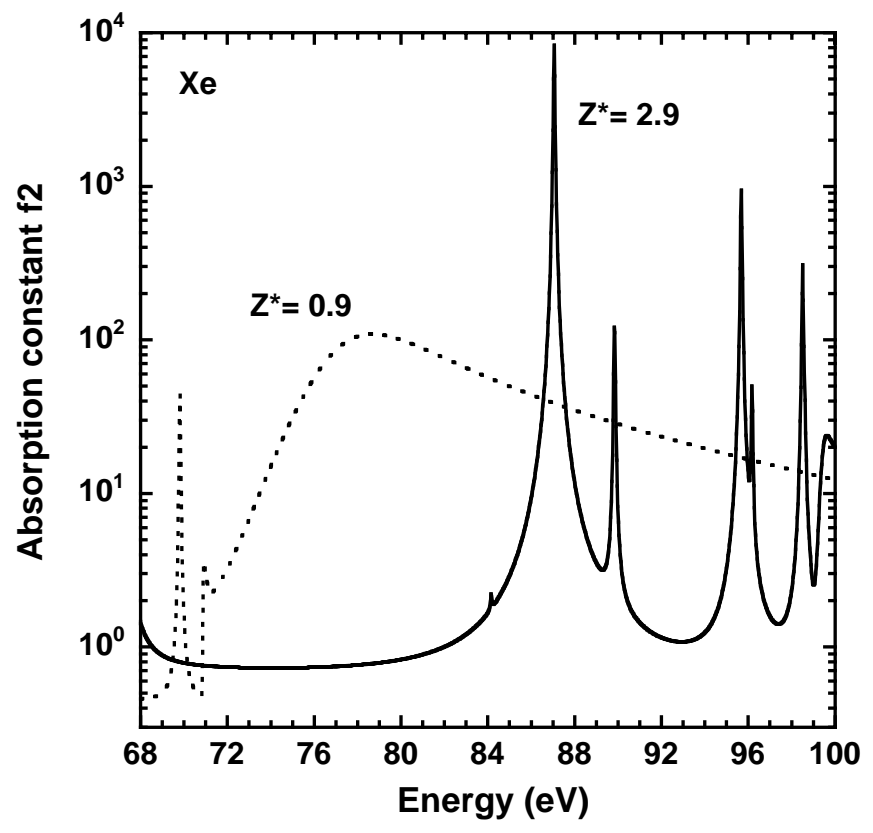

Fig. 5. Absorption constant $f_{2}$ versus photon energy for Xe plasmas. The average ionization state is given by $Z^{*}$. The dotted line is for $Z^{*}=0.9$ and the solid curve is for $Z^{*}=2.9$. The ion density is $10^{20} \mathrm{~cm}^{-3}$.

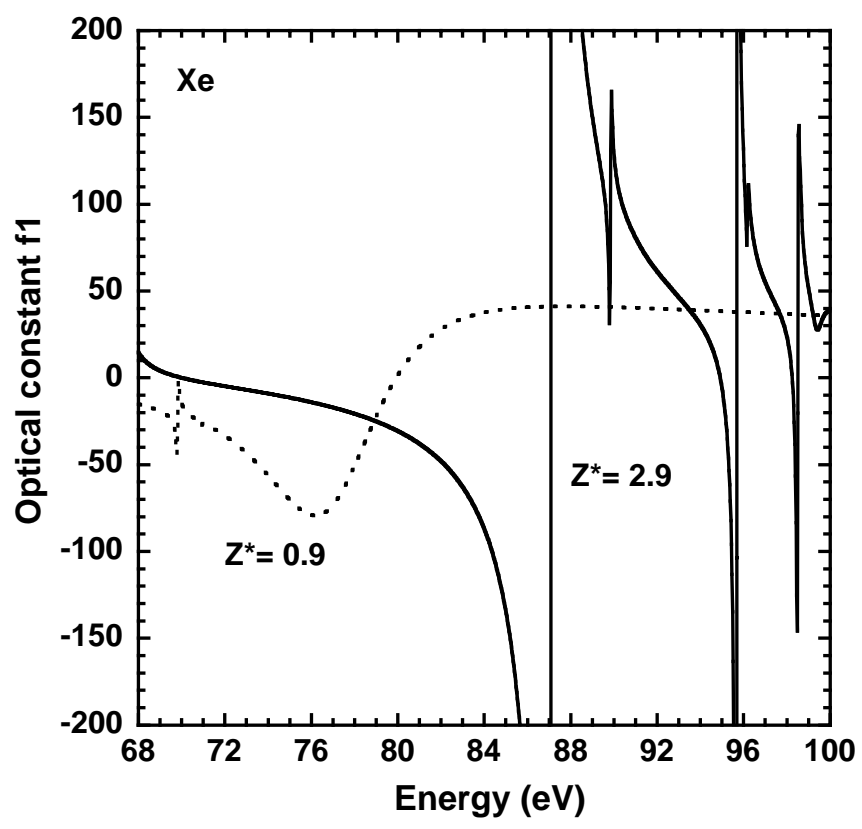

Fig. 6. Optical constant $f_{1}$ versus photon energy for Xe plasmas. The average ionization state is given by $Z^{*}$. The dotted line is for $Z^{*}=0.9$ and the solid curve is for $Z^{*}=2.9$. The ion density is $10^{20} \mathrm{~cm}^{-3}$. 


\section{MODELING OF XENON PLASMAS AT 14.7 NM}

The Henke tables show neutral Xe with a value of -9 for $\mathrm{f}_{1}$ at $14.7 \mathrm{~nm}(84.46 \mathrm{eV})$, the energy of the Ni-like Pd Xray laser. We have recently improved the average atom code so we can do calculations of materials like Xe. Xe is an interesting case because the oscillator strength for the $4 \mathrm{~d}$ state is primarily spread among the continuum photoionizations for neutral Xe through $\mathrm{Xe}^{2+}$ but suddenly collapses into the $4 \mathrm{~d}-4 \mathrm{f}$ transition as the $4 \mathrm{f}$ orbital becomes bound for $\mathrm{Xe}^{3+}$. Figure 5 shows the absorption constant $\mathrm{f}_{2}$ calculated by the average atom code for the cases of Xe with $\mathrm{Z}^{*}=$ 0.9 and $\mathrm{Z}^{*}=2.9$. The spectra are shifted by 2.5 and $8.5 \mathrm{eV}$, respectively, to agree better with the experimental data for $\mathrm{Xe}^{1+}$ and $\mathrm{Xe}^{3+}$. One observes how the absorption goes from being spread over 10 's of eV for the singly-ionized case $\left(Z^{*}=0.9\right)$ to being largely concentrated in the single $4 \mathrm{~d}-4 \mathrm{f}$ line at $87 \mathrm{eV}$ for the triply ionized case $\left(Z^{*}=2.9\right)$. In both cases the total oscillator strength associated with the $4 \mathrm{~d}$ level is about 10. If we now look at the optical constant $\mathrm{f}_{1}$, Fig. 6 shows how $f_{1}$ is a large negative value below the $87 \mathrm{eV}$ line for the case of $Z^{*}=2.9$ while for the $Z^{*}=0.9$ case $f_{1}$ is positive and the plasma is highly absorbing. Recently Erik Emmons and his colleagues did a excellent set of experiments [26] at the Advance Light Source (ALS) at Lawrence Berkeley Laboratory to measure the absorption coefficient of $\mathrm{Xe}^{+3}$ with high resolution $(0.05 \mathrm{eV})$ over the 37 to $117 \mathrm{eV}$ region. Emmons graciously provided us with their detailed data so that we could calculated the optical constant $f_{1}$ from the absorption spectrum for $\mathrm{Xe}^{3+}$. This is shown in Fig. 7. At $84.46 \mathrm{eV}, \mathrm{f}_{1}$ is -35 , which means there should be a tremendous negative fringe shift with the index of refraction larger than one. From 72 to $87 \mathrm{eV} \mathrm{f}_{1}$ is negative. This is primarily due to the very strong $4 \mathrm{~d}-4 \mathrm{f}$ line at 87.0 $\mathrm{eV}$. For the Ni-like Ag X-ray laser that lases at $13.9 \mathrm{~nm}(89.25 \mathrm{eV}) \mathrm{f}_{1}$ is 16 since the laser line is on the high-energy side of the $\mathrm{Xe}^{+3}$ absorption line. In this case the experiment would bend the normal direction but the experiment would overestimate the electron density by a factor of five. Fig. 7 helps validate our calculations shown in Fig. 6.

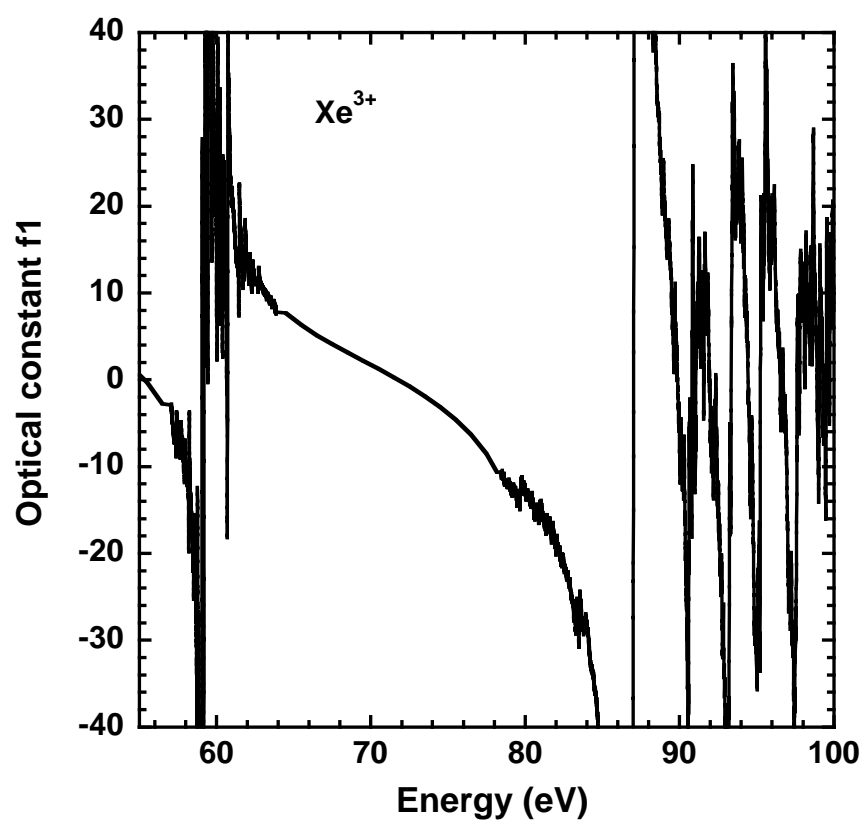

Fig. 7. Optical constant $\mathrm{f}_{1}$ versus photon energy for $\mathrm{Xe}^{3+}$ calculated from experimental data in Ref. 26. 


\section{CONCLUSIONS}

Since the earliest days of lasers the analysis of plasma diagnostics such as interferometers have relied on the approximation that the index of refraction in plasmas is due solely to the free electrons. This makes the index of refraction less than one and is an essential assumption used in determining the critical density surface for energy deposition in the plasma and for doing photon transport calculations. Recent X-ray laser interferometer measurements of $\mathrm{C}, \mathrm{Al}, \mathrm{Ag}$, and Sn plasmas have observed anomalous results with the index of refraction being greater than one. The analysis of these plasmas show that the anomalous dispersion from both the resonance lines and absorption edges due to the bound electrons can have the dominant contribution to the index of refraction. It is well known that a strong resonance line can cause anomalous results near the absorption line but this work shows that the effects from the resonance lines extend to photon energies located orders of magnitude further from the line centers than the corresponding line widths owing to the fact that they contribute through a dispersion integral. Similar long-range effects are shown for the absorption edges.

In this paper we search for other candidate materials that would have an index of refraction greater than one. Utilizing our average atom code we calculate the index of refraction in $\mathrm{Ne}, \mathrm{Na}$, and $\mathrm{Xe}$ plasmas, which we identified as candidate materials that would have anomalous dispersion for photon energies from the optical to $100 \mathrm{eV}$ soft X-rays. We show that singly-ionized neon and neutral sodium should have an index greater than one at the energy of the Ne-like Ar X-ray laser at $46.9 \mathrm{~nm}(26.44 \mathrm{eV})$. We also show that triply-ionized xenon looks like a promising candidate at the $84.46 \mathrm{eV}$ energy of the interferometer based on the Ni-like Pd X-ray laser. Finally we use recent high-resolution absorption measurements of triply-ionized xenon done at the ALS to validate our average atom calculations for Xe.

With the advent of the FLASH X-ray free electron laser in Germany and the LCLS X-FEL coming online at Stanford in 2 years the average atom code will be an invaluable tool to explore plasmas at higher X-ray energy to identify potential experiments for the future. During the next decade X-ray free electron lasers and other sources will be available to probe a wider variety of plasmas at higher densities and shorter wavelengths so it will be even more essential to understand the index of refraction in plasmas. X-ray laser interferometers may become a valuable tool to measure the index of refraction of plasmas in the future.

\section{ACKNOWLEDGEMENTS}

We wish to thank Erik Emmons for providing us detailed absorption data from the $\mathrm{Xe}^{+3}$ measurements done at the ALS. This work was performed under the auspices of the U. S. Department of Energy by the University of California Lawrence Livermore National Laboratory under contract No.W-7405-ENG-48. This research was also sponsored by the National Nuclear Security Administration under the Stewardship Science Academic Alliances program through U. S. Department of Energy Research Grant \# DE-FG52-06NA26152. The work of one author (WRJ) was supported in part by NSF Grant No. PHY-0456828. 


\section{REFERENCES}

[1] G. J. Tallents, J. Phys. D. 17, 721 (1984).

[2] H. R. Griem, Principles of Plasma Spectroscopy, (Cambridge University Press, Cambridge, 1997) p. 9

[3] L. B. Da Silva, T. W. Barbee, Jr., R. Cauble, P. Celliers, D. Ciarlo, S. Libby, R. A. London, D. Matthews, S. Mrowka, J. C. Moreno, D. Ress, J. E. Trebes, A. S. Wan, and F. Weber, Phys. Rev. Lett. 74, 3991 (1995).

[4] H. Tang, O. Guilbaud, G. Jamelot, D. Ros, A. Klisnick, D. Joyeux, D. Phalippou, M. Kado, M. Nishikino, M.

Kishimoto, K. Sukegawa, M. Ishino, K. Nagashima, and H. Daido, Appl. Phys. B 78, 975 (2004).

[5] J. Filevich, J. J. Rocca, M. C. Marconi, S. Moon, J. Nilsen, J. H. Scofield, J. Dunn, R. F. Smith, R. Keenan, J. R.

Hunter, and V. N. Shlyaptsev, Phys. Rev. Lett. 94, 035005 (2005).

[6] J. Filevich, K. Kanizay, M. C. Marconi, J. L. A. Chilla, and J. J. Rocca, Opt. Lett 25, 356 (2000).

[7] D. Descamps, C. Lyngå, J. Norin, A. L’Hullier, C.-G. Wahlström, J.-F. Hergott, H. Merdji, P. Salières, M. Bellini, and T. W. Hänsch, Opt. Lett. 25, 135 (2000).

[8] A. Meseck, M. Abo-Bakr, D. Krämer, B. Kuske, and S. Reiche, Nucl. Inst. And Meth. A 528, 577 (2004).

[9] J. Filevich, J. J. Rocca, M. C. Marconi, S. J. Moon, J. Nilsen, J. H. Scofield, J. Dunn, R. F. Smith, R. Keenan, J. R. Hunter, and V. N. Shlyaptsev, J. Quant. Spectrosc. Radiat. Transfer 99, 165 (2006).

[10] J. Filevich, J. Grava, M. Purvis, M. C. Marconi, J. J. Rocca, J. Nilsen, J. Dunn, W. R. Johnson, Phys. Rev. E 74, 016404 (2006).

[11] J. Filevich, J. Grava, M. Purvis, M. C. Marconi, J. J. Rocca, J. Nilsen, J. Dunn, W. R. Johnson, Laser and Particle Beams 25, 47 (2007).

[12] J. Nilsen and J. H. Scofield, Opt. Lett. 29, 2677 (2004).

[13] J. Nilsen, W. R. Johnson, C. A. Iglesias, and J. H. Scofield, J. Quant. Spectrosc. Radiat. Transfer 99, 425 (2006).

[14] J. Nilsen and W. R. Johnson, Appl. Opt. 44, 7295 (2005).

[15] D. A. Liberman, JQSRT 27, 335 (1982).

[16] W. R. Johnson, C. Guet, and G. F. Bertsch, J. Quant. Spectrosc. Radiat. Transfer 99, 327 (2006).

[17] C. A. Iglesias, F. J. Rogers, and B. G. Wilson, Astrophys. J. Lett. 322, L45 (1987).

[18] F. J. Rogers, B. G. Wilson, and C. A. Iglesias, Phys. Rev. A 38, 5007 (1988).

[19] C. A. Iglesias and F. J. Rogers Astrophys. J. 464, 943 (1996).

[20] B. L. Henke, E. M. Gullikson, and J. C. Davis, ADNDT 54, 181 - 342 (1993).

[21] L. D. Landau and E. M. Lifshitz, Electrodynamics of Continuous Media, (Pergamon, New York, 1984) pp. 280 283

[22] D. A. Greenwood, Proc. Phys. Soc. London 715, 585 (1958).

[23] R. Kubo, J. Phys. Soc. Jpn. 12, 570 (1957).

[24] W. Persson, Phys. Scr. 3, 133 (1971).

[25] T. Lundstrom and L. Minnhagen, Phys. Scr. 5, 243 (1972).

[26] E. D. Emmons, A. Aguilar, M. F. Gharaibeh, S. W. J. Scully, R. A. Phaneuf, A. L. D. Kilcoyne, A. S. Schlachter, I. Àlverez, C. Cisneros, and G. Hinojosa, Phys. Rev. A 71, 042704 (2005). 